\title{
The diffuse molecular component in the nuclear bulge of the Milky Way ${ }^{\star}$
}

\author{
D. Riquelme ${ }^{1, \star \star}$, L. Bronfman ${ }^{2}$, R. Mauersberger ${ }^{1,2}$, R. Finger $^{2}$, C. Henkel ${ }^{1,3}$, T. L. Wilson ${ }^{4}$, and P. Cortés-Zuleta ${ }^{2}$ \\ 1 Max-Planck-Institut für Radioastronomie, Auf dem Hügel 69, 53121 Bonn, Germany \\ e-mail: riquelme@mpifr-bonn.mpg.de \\ 2 Departamento de Astronomía, Universidad de Chile, Casilla 36-D, Santiago, Chile \\ 3 Astronomy Department King Abdulaziz University, PO Box 80203, 21589 Jeddah, Saudi Arabia \\ ${ }^{4}$ NSF Division of Astronomical Sciences. Suite 1053, 4201 Wilson Blvd., Arlington, VA 22230, USA \\ Received 10 February 2017 / Accepted 4 September 2017
}

\begin{abstract}
Context. The bulk of the molecular gas in the central molecular zone (CMZ) of the Galactic center region shows warm kinetic temperatures, ranging from $>20 \mathrm{~K}$ in the coldest and densest regions $\left(n \sim 10^{4-5} \mathrm{~cm}^{-3}\right)$ up to more than $100 \mathrm{~K}$ for densities of about $n \sim 10^{3} \mathrm{~cm}^{-3}$. Recently, a more diffuse, hotter $\left(n \sim 100 \mathrm{~cm}^{-3}, T \sim 250 \mathrm{~K}\right)$ gas component was discovered through absorption observations of $\mathrm{H}_{3}^{+}$. This component may be widespread in the Galactic center, and low density gas detectable in absorption may be present even outside the CMZ along sightlines crossing the extended bulge of the Galaxy.

Aims. We aim to observe and characterize diffuse and low density gas using observations of 3-mm molecular transitions seen in absorption.

Methods. Using the Atacama Large (sub)Millimeter Array (ALMA) we observed the absorption against the quasar J1744-312, which is located toward the Galactic bulge region at $(l, b)=(-2: 13,-1: 0)$, but outside the main molecular complexes.

Results. ALMA observations in absorption against the J1744-312 quasar reveal a rich and complex chemistry in low density molecular and presumably diffuse clouds. We detected three velocity components at $\sim 0,-153$, and $-192 \mathrm{~km} \mathrm{~s}^{-1}$. The component at $\sim 0 \mathrm{~km} \mathrm{~s}{ }^{-1}$ could represent gas in the Galactic disk while the velocity components at -153 , and $-192 \mathrm{~km} \mathrm{~s}^{-1}$ likely originate from the Galactic bulge. We detected 12 molecules in the survey, but only 7 in the Galactic bulge gas.
\end{abstract}

Key words. ISM: abundances - ISM: clouds - ISM: molecules - ISM: kinematics and dynamics - Galaxy: center - radio lines: ISM

\section{Introduction}

Until recently, our view of the interstellar medium (ISM) of the central molecular zone (CMZ; i.e., the innermost $500 \mathrm{pc}$ ) of the Milky Way was that it consists of basically three phases: an ionized and low density gas component $\left(T \sim 10^{4-6} \mathrm{~K}, n \sim 10 \mathrm{~cm}^{-3}\right)$ responsible for the well-studied fine structure and radio recombination line emission; an ultra high-temperature X-ray-emitting plasma that covers a large portion of the CMZ, and dense and warm giant molecular clouds, which amount to about $10^{7} M_{\odot}$ and contain $\sim 80 \%$ of the dense gas (Morris \& Serabyn 1996; Lazio \& Cordes 1998; Geballe et al. 2011; Ferrière et al. 2007). This molecular phase shows warm gas with kinetic temperatures ranging from $\sim 20 \mathrm{~K}$ in the coldest and densest regions $\left(n \sim 10^{4-5} \mathrm{~cm}^{-3}\right.$ ) up to more than $100 \mathrm{~K}$ when densities decrease to $n \sim 10^{3} \mathrm{~cm}^{-3}$ (Hüttemeister et al. 1993). Recently, using $\mathrm{H}_{2} \mathrm{CO}$ observations, Ginsburg et al. (2016) showed that warm $(50-120 \mathrm{~K})$ and dense gas $\left(n \sim 10^{4-5} \mathrm{~cm}^{-3}\right)$ pervades the CMZ. However, detections of $\mathrm{H}_{3}^{+}$absorption (Goto et al. 2002) led to the discovery of a vast amount of high-temperature

* This paper makes use of the following ALMA data: ADS/JAO.ALMA\#2012.1.00119.S. ALMA is a partnership of ESO (representing its member states), NSF (USA) and NINS (Japan), together with NRC (Canada), NSC and ASIAA (Taiwan), and KASI (Republic of Korea), in cooperation with the Republic of Chile. The Joint ALMA Observatory is operated by ESO, AUI/NRAO, and NAOJ. $\star \star$ This work was partially carried out during several visits of the principal author to the Astronomy Department of the Universidad de Chile.
$(T \sim 250 \mathrm{~K})$ and low density $\left(n \sim 100 \mathrm{~cm}^{-3}\right)$ gas with a high velocity dispersion in the CMZ (Oka et al. 2005; Goto et al. 2008; Geballe \& Oka 2010; Le Petit et al. 2016). This new component presumably represents a diffuse molecular medium that is widespread in the CMZ and distinct from the three ISM components already known. Although the absorbing gas seems to be ubiquitous toward the Galactic center (GC) region, little is known about its volume filling factor, mass, the detailed excitation conditions $\left(n\left(\mathrm{H}_{2}\right), T_{\text {kin }}\right)$, chemical composition, and the extension of the diffuse molecular medium. This diffuse molecular component has been detected very recently by Gerin \& Liszt (2017) in three species, namely $\mathrm{HCO}^{+}, \mathrm{HCN}$, and $\mathrm{HNC}$.

The new component largely escaped detection in large scale surveys in the $\mathrm{cm}$ and $\mathrm{mm}$ range because molecules require a sufficient abundance in the upper energy levels and a dipole moment to be detectable in emission. $\mathrm{CO}$ is often used as a tracer of $\mathrm{H}_{2}$ since it has a dipole moment, even a sufficiently small dipole moment such that its rotational levels can be populated by collisions with $\mathrm{H}_{2}$ when densities are less than $1000 \mathrm{~cm}^{-3}$. However, when densities are lower than a few $100 \mathrm{~cm}^{-3}$ and the excitation temperature of the even lower $\mathrm{CO}$ rotational transitions comes close to the cosmic microwave background, the emission from $\mathrm{CO}$ (and other molecules) becomes completely invisible because of the lack of excitation above the cosmic microwave background (CMB) emission.

Fortunately, one can detect such low excitation gas, namely via its absorption lines. Toward the disk of the Milky Way, for 


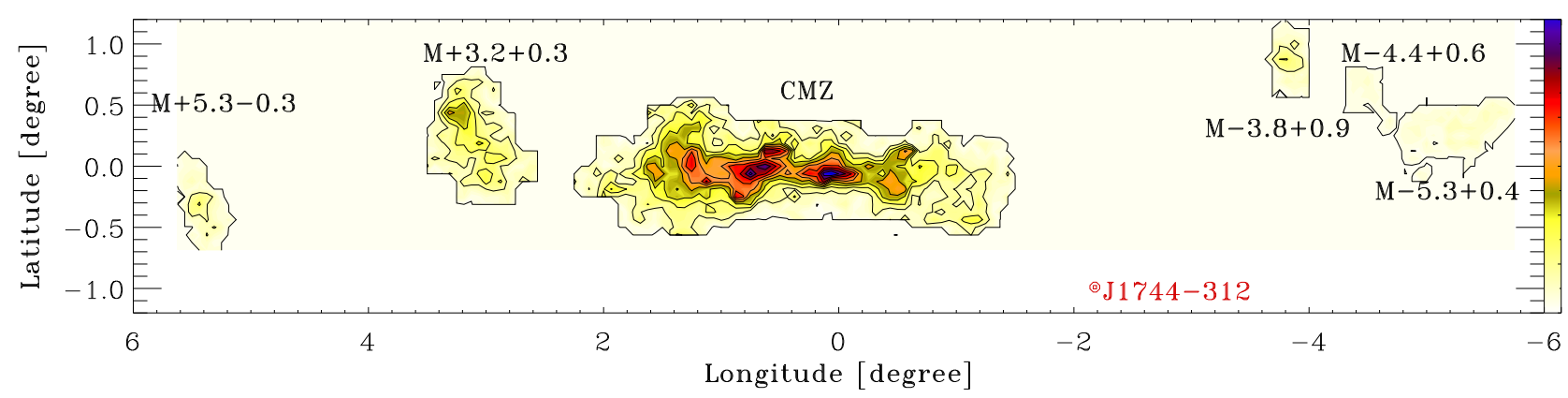

Fig. 1. Overview of the Galactic center region in the $\mathrm{HCO}^{+}(1-0)$ line from Riquelme et al. (2010b). The position of the quasar J1744-312 is indicated.

example, one can trace diffuse and translucent gas by looking for absorption lines toward quasars that happen to be within 20 degrees of the Galactic plane (Liszt \& Lucas 2001). A surprisingly complex polyatomic chemistry exists in such diffuse clouds, allowing for detections of species such as $\mathrm{C}_{2} \mathrm{H}, \mathrm{C}_{3} \mathrm{H}_{2}$, $\mathrm{H}_{2} \mathrm{CO}$, and $\mathrm{NH}_{3}$, which have relative abundances that are strikingly similar to those of the Taurus molecular cloud 1 (TMC-1; Liszt et al. 2008). In extragalactic studies, absorption against the quasar PKS1830-211 reveals a rich chemistry in the disk of a galaxy at $z=0.89$ (Muller et al. 2011, 2016; Menten et al. 1999, 2008). Muller et al. (2011) measured many absorption lines and found excitation temperatures of almost all species involved around $5.1 \mathrm{~K}$, i.e., the $\mathrm{CMB}$ temperature at that distance. These lines would therefore be impossible to detect in emission. Henkel et al. (2008) showed from $\mathrm{NH}_{3}$ absorption lines toward the same quasar that despite the low excitation, the kinetic temperature of the gas ranges from $T=80$ up to $600 \mathrm{~K}$, and may therefore be similar in excitation conditions to the component claimed by Geballe \& Oka (2010) for our GC region. Also toward the $\mathrm{CMZ}$, ammonia absorption against Sgr B2 reveals low excitation gas with a kinetic temperature exceeding $1300 \mathrm{~K}$ (Wilson et al. 2006). Wilson et al. (2006) assumed that this hot gas is physically associated with the active region Sgr B2. Following the claim by Geballe \& Oka (2010), it may well be that what Wilson et al. observed is indeed hot, low excitation, and most importantly, diffuse and widespread inter-cloud gas in the $\mathrm{GC}$ region.

Here we intend to search for the presence of the low density gas by looking for absorption toward a source along a sightline beyond the CMZ that is crossing the bulge of the Galaxy and is not associated with a giant molecular cloud. There are still many unknowns about this new component such as excitation condition and molecular composition. Therefore we carried out a pilot study to detect molecular absorption against the quasar J1744-312 located behind the bulge of the Galaxy in order to confirm and characterize the diffuse molecular component in the GC region proposed by Geballe \& Oka (2010).

We chose the quasar J1744-312 as the background source to look for the absorption components in the GC region. J1744-312 is at $(l, b)=(-2.13,-1.00)$, i.e., behind the Galactic bulge ${ }^{1}$. Figure 1 shows the position of the quasar in the GC region. This quasar is compact since it is at best marginally resolved with a $4.5 \times 1.1$ mas beam at $24 \mathrm{GHz}$ (B1741-312 in Charlot et al. 2010), and it is expected that the quasar is even more compact at mm-wavelengths. In addition interstellar scintillation, which can result in a broadening of the source size, is negligible in

\footnotetext{
1 Throughout this work, we refer as the Galactic center region to the central kpc of the Galaxy, which is shown in Fig. 1 and is related to the bulge of the Galaxy.
}

our case since the quasar is far from the CMZ and scintillation becomes less visible at mm-wavelengths. The quasar has a flux density of $0.571 \mathrm{Jy}$ at $103 \mathrm{GHz}$ and $0.563 \mathrm{Jy}$ at $91.5 \mathrm{GHz}$ in the ALMA-Band 3 calibrator source catalog (see the ALMA Calibrator Source Catalogue ${ }^{2}$ ); this quasar is therefore an adequate source for absorption measurements of gas in the Galactic bulge. Unlike the absorption detected by Geballe \& Oka (2010), this quasar is not behind one of the prominent molecular complexes. This may serve to avoid confusion with absorption by denser gas components.

\section{Observations}

The observations were carried out during the ALMA Early Science Cycle 1 (ALMA project 2012.1.00119.S) using 29 12-m antennas during five observing periods (May 02; Jun. 29; Jul. 01, $02,03$, and 21,2014$)$. The coordinates for the quasar used for the observations were $\alpha(\mathrm{J} 2000): 17^{\mathrm{h}} 44^{\mathrm{m}} 23.5782^{\mathrm{s}}, \delta(\mathrm{J} 2000)$ : -31 deg $16^{\prime} 36^{\prime \prime}$.287. We performed a spectral survey of the ALMA band 3, covering a frequency range from 84.114 to $114.616 \mathrm{GHz}$ using five spectral setups, each of these covering $4 \times 1.875 \mathrm{GHz}$ bandwidth with a channel separation of $2.584 \mathrm{MHz}$ corresponding to $\sim 10 \mathrm{~km} \mathrm{~s}^{-1}$. The primary beam full width at half maximum (FWHM) size ranges from 73.5 arcsec at $84.114 \mathrm{GHz}$ to $54 \mathrm{arcsec}$ at $114.616 \mathrm{GHz}$. Table 1 shows the details of the observations and the sensitivity reached in each setup. The calibration was performed by the ALMA team at the North America Arc node.

\section{Results and analysis}

The analysis of the data was carried out with the Greg and Class packages of the GILDAs software ${ }^{3}$. Our data consist of absorption lines and continuum levels toward the quasar. We extracted a single spectrum toward the center of the quasar. The continuum level ranges from 0.55 to $0.70 \mathrm{Jy}$ with small variations not only along the band $(<5 \%)$, but also between the different observing periods $(<14 \%)$, which suggests intrinsic intensity variations of J1744-312. Then, to compute the optical depth, we estimated the line-to-continuum ratio for each detected line (see Sect. 3.2).

\subsection{Line identification and line fit}

Figure 2 shows the complete spectrum normalized to the detected continuum level. The line identification was carried out with the Cologne Database for Molecular Spectroscopy (CDMS)

\footnotetext{
2 https://almascience.eso.org/sc/

3 http://www.iram. fr/IRAMFR/GILDAS
} 
Table 1. Observational setups.

\begin{tabular}{|c|c|c|c|c|c|c|c|c|}
\hline Setup & $\begin{array}{l}\text { Spectral } \\
\text { window }\end{array}$ & $\begin{array}{c}\text { Frequency range } \\
\mathrm{GHz}\end{array}$ & $\begin{array}{c}\text { Central frequency } \\
\mathrm{GHz}\end{array}$ & $\begin{array}{l}\mathrm{rms} \\
\mathrm{mJy}\end{array}$ & $\begin{array}{l}\text { Velocity } \\
\text { resolution } \\
\mathrm{km} \mathrm{s}^{-1}\end{array}$ & $\begin{array}{l}\text { Primary } \\
\text { beam } \\
\text { arcsec }\end{array}$ & $\begin{array}{c}\text { Synthesized } \\
\text { beam } \\
\text { arcsec }\end{array}$ & Observation date \\
\hline \multirow[t]{4}{*}{1} & 0 & $91.483-93.355$ & 92.416 & 2.56 & 8.42 & 66.87 & $1.55 \times 1.36$ & 2014-06-29 \\
\hline & 1 & $93.316-95.188$ & 94.264 & 2.37 & 8.42 & 65.56 & $1.45 \times 1.34$ & \\
\hline & 2 & $103.627-105.498$ & 104.563 & 2.59 & 8.42 & 59.10 & $1.34 \times 1.24$ & \\
\hline & 3 & $105.483-107.354$ & 106.412 & 2.56 & 8.42 & 58.07 & $1.35 \times 1.23$ & \\
\hline \multirow[t]{4}{*}{2} & 0 & $95.158-97.029$ & 96.090 & 3.19 & 8.13 & 64.31 & $1.91 \times 1.35$ & 2014-07-01 \\
\hline & 1 & $96.991-98.860$ & 97.934 & 3.20 & 8.13 & 63.10 & $1.87 \times 1.36$ & \\
\hline & 2 & $107.303-109.173$ & 108.240 & 3.77 & 8.13 & 57.09 & $1.74 \times 1.31$ & \\
\hline & 3 & $109.158-111.030$ & 110.090 & 3.83 & 8.13 & 56.14 & $1.70 \times 1.28$ & \\
\hline \multirow[t]{4}{*}{3} & 0 & $87.810-89.681$ & 88.742 & 2.94 & 8.73 & 69.64 & $1.80 \times 1.49$ & 2014-07-03 \\
\hline & 1 & $89.643-91.513$ & 90.590 & 3.19 & 8.73 & 68.22 & $1.75 \times 1.50$ & \\
\hline & 2 & $99.954-101.825$ & 100.890 & 3.05 & 8.73 & 61.25 & $1.63 \times 1.43$ & \\
\hline & 3 & $101.810-103.680$ & 102.740 & 3.24 & 8.73 & 60.15 & $1.61 \times 1.41$ & \\
\hline \multirow[t]{4}{*}{4} & 0 & $110.925-112.798$ & 111.861 & 2.99 & 8.83 & 55.25 & $1.55 \times 1.26$ & 2014-07-02 \\
\hline & 1 & $112.747-114.616$ & 113.695 & 4.01 & 8.83 & 54.36 & $1.53 \times 1.23$ & \\
\hline & 2 & $98.789-100.660$ & 99.714 & 2.46 & 8.83 & 61.98 & $1.76 \times 1.36$ & \\
\hline & 3 & $100.611-102.480$ & 101.547 & 2.32 & 8.83 & 60.86 & $1.63 \times 1.33$ & \\
\hline \multirow[t]{4}{*}{5} & 0 & $84.114-85.994$ & 85.067 & 1.35 & 12.04 & 72.65 & $1.70 \times 1.40$ & 2014-05-02, 2014-07-21 \\
\hline & 1 & $85.970-87.850$ & 86.916 & 1.47 & 11.79 & 71.10 & $1.65 \times 1.41$ & \\
\hline & 2 & $96.280-98.160$ & 97.216 & 1.53 & 10.54 & 63.57 & $1.56 \times 1.33$ & \\
\hline & 3 & $98.113-99.993$ & 99.064 & 1.56 & 10.34 & 62.38 & $1.51 \times 1.33$ & \\
\hline
\end{tabular}

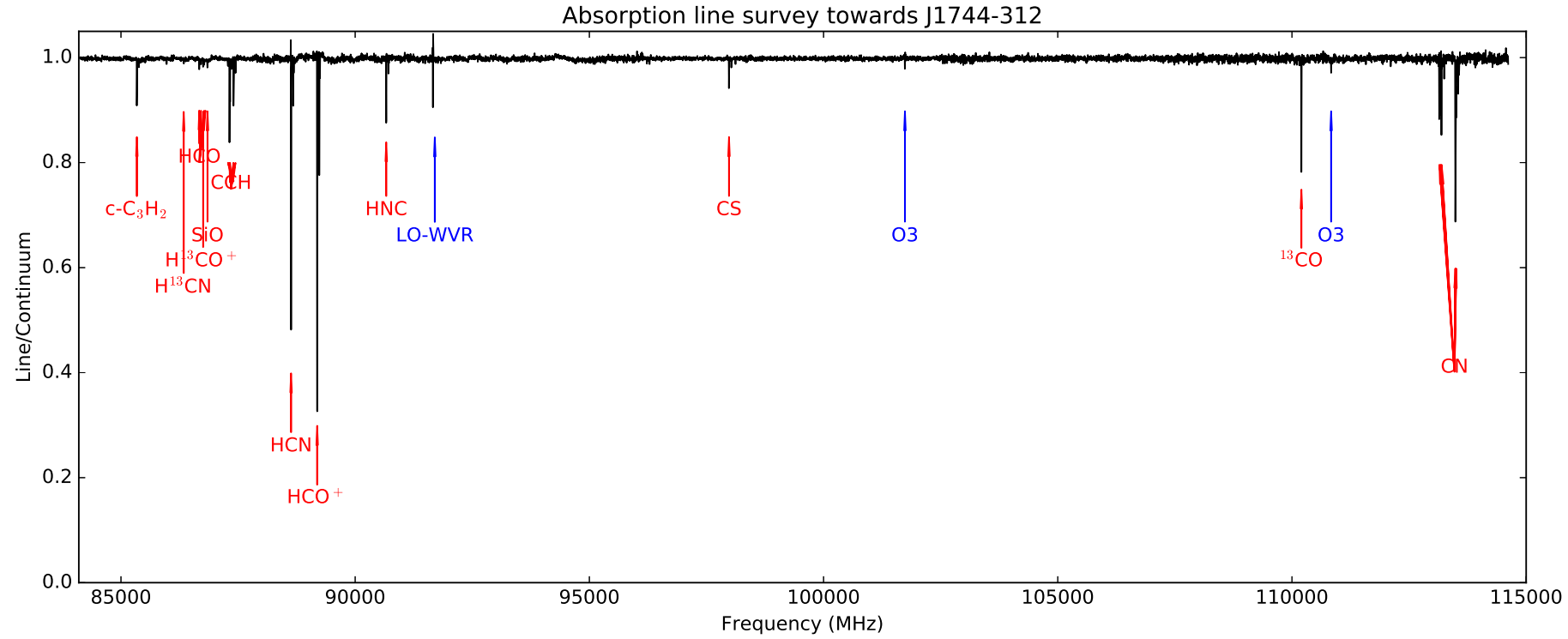

Fig. 2. Three-mm survey toward J1744-312. The signal was normalized to the continuum level. In red all detected interstellar molecular transitions are shown. In blue we show the atmospheric $\mathrm{O}_{3}$ lines and interference by the local oscillators of the water vapor radiometers (see Sect. 3.1).

catalog (Müller et al. 2005, 2001) and the Jet Propulsion Laboratory Molecular Spectroscopy (JPL) catalog (Pickett et al. 1998). Twelve species were detected, including three molecules with hyperfine structure (HFS), namely $\mathrm{HCO}, \mathrm{CN}$, and $\mathrm{CCH}^{4}$; one instrumental artifact at $91.7 \mathrm{GHz}$, and two atmospheric transitions at 101.74 and $110.836 \mathrm{GHz}$. Table A.1 shows the spectral parameters of all detected interstellar molecular transitions.

The features close to $91.65 \mathrm{GHz}$, which are seen in emission and absorption, can be ascribed to interference by the local oscillators (LO) of the water vapor radiometers (WVR) that each of the ALMA antennas is equipped with (Nikolic et al.

\footnotetext{
$4 \mathrm{HCN}$ also have HFS but this is not resolved because of poor spectral resolution.
}

2013). The signal of the LO, if insufficiently shielded, may be picked up by one or several neighboring ALMA antennas. Even though each LO in the various WVRs is tuned to a slightly different frequency around $91.7 \mathrm{GHz}$ to avoid a correlated product, this measure is apparently not sufficient to prevent an interference free signal at a frequency close to $91.7 \mathrm{GHz}$. The features at 110.836 and $101.74 \mathrm{GHz}$ correspond to atmospheric $\mathrm{O}_{3}$ (Shimabukuro \& Wilson 1973; Caton et al. 1968).

In order to characterize the absorption profile with a higher signal-to-noise level, we averaged spectra of the four strongest transitions that do not have HFS. This stacked spectrum clearly shows three velocity components at $\sim 0,-153$, and $-192 \mathrm{~km} \mathrm{~s}^{-1}$ (see Fig. 3). The component at $\sim 0 \mathrm{~km} \mathrm{~s}^{-1}$ could correspond to 


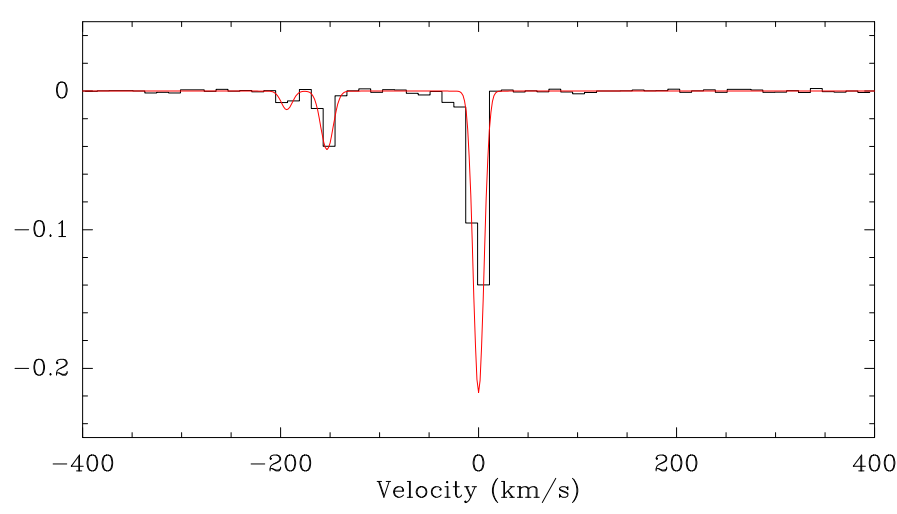

Fig. 3. Stacked spectrum of the four strongest transitions that show the three velocity components and do not have hyperfine structure $\left(\mathrm{HCO}^{+}\right.$, $\mathrm{HNC}, \mathrm{CS}, \mathrm{c}-\mathrm{C}_{3} \mathrm{H}_{2}$ ).

gas in the Galactic disk, and we argue in Sect. 4.1 that the velocity components at -153 and $-192 \mathrm{~km} \mathrm{~s}^{-1}$ are from the bulge of the Galaxy. The absorption profiles were fitted with Gaussian functions (Table 2). The intensity peak is negative because the fits are performed on the continuum subtracted spectra. The appendix (Figs. A.1 and A.2) shows Gaussian fits for each detected species. Because of the low velocity resolution of the observations, the fits did not always converge and in those cases we fixed the linewidths to the value given by the Gaussian fit isolating each corresponding velocity component (Table 2 ). We clearly detected four HFS components of HCO, six HFS components of $\mathrm{CCH}$, and seven of the nine HFS components of CN. These lines were modeled with the HFS method of CLASS assuming optically thin emission and the same excitation temperature and linewidth for all components of the multiplet. The relative intensities of the HFS of these molecules are shown in Table 3. Only the velocity component at $0 \mathrm{~km} \mathrm{~s}^{-1}$ was detected in $\mathrm{HCO}$. Two velocity components were detected in $\mathrm{CN}$ and $\mathrm{CCH}$. The component at $\sim 0 \mathrm{~km} \mathrm{~s}^{-1}$ is shown in red in Figs. A.1 and A.2, and the velocity component at $\sim-153 \mathrm{~km} \mathrm{~s}^{-1}$ is shown in blue. The main HFS component is used to determine the total optical depth for $\mathrm{HCO}$ and $\mathrm{CCH}$ because they are not blended with any other species. CN shows a more complex spectrum with three HFS features blended close to the main component. Here the $\mathrm{CN}$ line at $113.191 \mathrm{GHz}$, which is not blended with any other HFS feature (see Table 3 and Fig. A.2), was used to determine the column densities (see Sect. 3.2)

We compared the observed with the predicted line intensity ( $S_{1}$, flux density peak) ratios of different HFS components in the optically thin case under local thermodynamic equilibrium (LTE) conditions (Table 2). For $\mathrm{CCH}$, the theoretical line ratio between the main line at $87.31689 \mathrm{GHz}$ and the satellites at 87.32858 and $87.40198 \mathrm{GHz}$, which have the same relative intensity of 0.208 , (see Table 2) is 2.0 and the measured line intensity ratios (Gaussian peaks) are 1.9 and 2.0, respectively. For $\mathrm{HCO}$, the theoretical line ratio between the main line at $86.67076 \mathrm{GHz}$ and the satellites at 86.70836 and $86.77746 \mathrm{GHz}$ (both with the same relative intensity) is 1.7 , while the measured line intensity ratios are 2.0 and 1.8, respectively. For $\mathrm{CN}$, the main line is blended with other satellites; then, to compute the ratio, we used the line at $113.19127 \mathrm{GHz}$ with respect to the lines at $113.17049 \mathrm{GHz}$ and $113.14415 \mathrm{GHz}$, which have a theoretical line ratio of 1.3 and a measured line intensity ratio of 1.3 and 1.2, respectively. These results indicate that we can assume that $\mathrm{CCH}, \mathrm{CN}$, and $\mathrm{HCO}$ are optically thin following LTE conditions, and that our estimation of the column densities is reliable under this approach. Therefore, the determination of the optical depth and then, the column density, was carried out using only the main HFS component for $\mathrm{HCO}$ and $\mathrm{CCH}$, and the satellite at $113.191 \mathrm{GHz}$ for $\mathrm{CN}$, multiplying the obtained values by the proper factor (assumed: local thermodynamical equilibrium, optically thin absorption; see Sect. 3.2) to account for the other HFS features as well. The corresponding molecular parameters are shown in Table A.1.

In the spectra of some of the stronger lines $\left(\mathrm{HCN}, \mathrm{HCO}^{+}\right.$and $\mathrm{CN})$ there is a one channel wide emission adjacent to the absorption. This is most probably an artifact due to the correlation of steep spectral features, known as Gibbs ringing (Rupen 1999).

\subsection{Physical properties}

The derivation of the column density from absorption lines, from single dish observations, has been discussed by Linke et al. (1981) and Nyman (1984). Here we uses ALMA, the most powerful (sub-)mm synthesis instrument, to take point source absorption spectra toward a target with a $\sim 0.6$ flux density and milliarcsec size, to thus obtain accurate optical depth measurements. More recent derivations of column density from absorption lines data, using ALMA observations, can be found in Ando et al. (2016) and Muller et al. (2014).

Realistically assuming that the difference between the excitation temperature and that of the $\mathrm{CMB}$ is small in relation to the brightness temperature of the background quasar (actually, not only the difference but also $T_{\text {ex }}$ and $T_{\text {bg }}$ themselves are small with respect to $\left.T_{\text {quasar }}\right)$ and that the absorption covers the entire background source, the optical depth $(\tau)$ is related to the line/continuum flux density ratio by

$\tau=-\ln \left(1+\frac{S_{1}}{S_{\mathrm{c}}}\right)$

where $S_{1}$ is the flux density peak of the absorption line measured from the continuum level, and $S_{\mathrm{c}}$ is the flux density of the background continuum source. If all the molecular lines are spectroscopically resolved, then the $\tau$ estimated in this way is a good determination for the optical depth at the line peak.

However, even in the case of low spectral resolution, the line equivalent width (velocity integrated opacity in the optically thin case) is preserved and the total column density can be obtained with

$N=\frac{8 \pi v^{3}}{c^{3} A_{u l} g_{\mathrm{u}}} \frac{Q\left(T_{\mathrm{ex}}\right) \exp \frac{E_{1}}{k_{B} T_{\mathrm{ex}}}}{\left[1-\exp \left(\frac{-h v}{k_{B} T_{\mathrm{ex}}}\right)\right]} \int \tau \mathrm{d} V\left[\mathrm{~cm}^{-2}\right]$,

$k$ is the Boltzmann constant, $v$ the frequency of the transition, $h$ the Planck constant, $Q(T)$ the partition function at the assumed excitation temperature, $g_{\mathrm{u}}$ the upper state degeneracy, $A_{i j}$ the Einstein coefficient, and $E_{1}$ the energy of the lower level.

Table 2 shows the results for all the absorption features detected in the survey. As mentioned in Sect. 3.1, Gaussian fits were used; then, in Eq. (2), $\int \tau \mathrm{d} V=\sqrt{\frac{\pi}{4 \ln (2)}} \tau \tau_{\text {peak }} \Delta V$, where $\tau_{\text {peak }}$ is the $\tau$ at the peak of the absorption fitted by the Gaussian. Since the HFS line ratios are compatible with the theoretical LTE optically thin values (see Sect. 3.1), opacities (e.g., due to spectrally unresolved narrow features) cannot be much larger than those given in Table 2. This in turn makes clear that the background source coverage factor must indeed be close to unity. 
D. Riquelme et al.: The diffuse molecular component in the nuclear bulge of the Milky Way

Table 2. Line parameters and fitting results for the detected species.

\begin{tabular}{|c|c|c|c|c|c|c|c|c|}
\hline Molecule & $\begin{array}{l}\text { Rest. frequency } \\
\mathrm{GHz}\end{array}$ & $\begin{array}{l}V_{0}(\mathrm{LSR}) \\
\left(\mathrm{km} \mathrm{s}^{-1}\right)\end{array}$ & $\begin{array}{c}\Delta V \\
\left(\mathrm{~km} \mathrm{~s}^{-1}\right)\end{array}$ & $\begin{array}{l}S_{1} \\
\text { Jy }\end{array}$ & $\begin{array}{l}S_{\mathrm{c}} \\
\mathrm{Jy}\end{array}$ & $\begin{array}{l}\mathrm{rms} \\
\mathrm{mJy}\end{array}$ & $\tau$ & $\begin{array}{c}N \\
\left(\mathrm{~cm}^{-2}\right)\end{array}$ \\
\hline $\mathrm{c}-\mathrm{C}_{3} \mathrm{H}_{2}$ & 85.34 & $\begin{array}{c}-1.0(0.2) \\
-153.1(2.2) \\
-191.9(2.1)\end{array}$ & $\begin{array}{l}18.6^{a} \\
15.0^{a} \\
12.0^{a}\end{array}$ & $\begin{array}{l}-0.06615 \\
-0.00925 \\
-0.00583\end{array}$ & $\begin{array}{l}0.6195 \\
0.6195 \\
0.6195\end{array}$ & $\begin{array}{l}1.244 \\
1.244 \\
1.244\end{array}$ & $\begin{array}{l}0.113(0.012) \\
0.015(0.002) \\
0.009(0.001)\end{array}$ & $\begin{array}{l}1.362(0.400) \times 10^{13} \\
1.464(0.512) \times 10^{12} \\
7.362(3.261) \times 10^{11}\end{array}$ \\
\hline $\mathrm{H}^{13} \mathrm{CN}$ & 86.34 & $\begin{array}{c}-2.0(2.8) \\
- \\
-\end{array}$ & $\begin{array}{c}26.7(6.3) \\
- \\
-\end{array}$ & $\begin{array}{c}-0.00535 \\
- \\
-\end{array}$ & 0.6165 & 1.228 & $0.009(0.001)$ & $4.860(1.395) \times 10^{11}$ \\
\hline $\mathrm{HCO}^{b}$ & 86.71 & $\begin{array}{c}-0.2(0.8) \\
- \\
-\end{array}$ & $19.7(0.5)$ & $-0.01175^{b}$ & 0.6238 & 1.244 & $0.019(0.002)$ & $4.117(4.220) \times 10^{12}$ \\
\hline $\mathrm{H}^{13} \mathrm{CO}^{+}$ & 86.75 & $\begin{array}{c}0.2(1.3) \\
- \\
-\end{array}$ & $\begin{array}{c}22.7(3.8) \\
- \\
-\end{array}$ & -0.0101 & 0.62429 & 1.095 & $0.016(0.002)$ & $4.538(0.882) \times 10^{11}$ \\
\hline $\mathrm{SiO}$ & 86.85 & $2.4(2.4)$ & $22.7(7.2)$ & -0.00965 & 0.62401 & 1.103 & $0.016(0.002)$ & $1.283(0.427) \times 10^{12}$ \\
\hline $\mathrm{CCH}^{b}$ & 87.33 & $\begin{array}{c}-1.6(0.8) \\
-154.4(1.5)\end{array}$ & $\begin{array}{l}18.499(2.6) \\
26.680(4.6)\end{array}$ & $\begin{array}{l}-0.11309 \\
-0.01248\end{array}$ & $\begin{array}{l}0.62660 \\
0.62660\end{array}$ & $\begin{array}{l}1.260 \\
1.260\end{array}$ & $\begin{array}{l}0.199(0.021) \\
0.020(0.002)\end{array}$ & $\begin{array}{l}2.744(0.480) \times 10^{14} \\
4.000(0.795) \times 10^{13}\end{array}$ \\
\hline $\mathrm{HCN}$ & 88.63 & $\begin{array}{c}0.5(0.1) \\
-154.2(0.4) \\
-193.3(1.2)\end{array}$ & $\begin{array}{l}21.0(0.1) \\
10.8(1.1) \\
10.3(2.0)\end{array}$ & $\begin{array}{l}-0.39488 \\
-0.08517 \\
-0.03140\end{array}$ & $\begin{array}{l}0.65866 \\
0.65866 \\
0.65866\end{array}$ & $\begin{array}{l}2.953 \\
2.953 \\
2.953\end{array}$ & $\begin{array}{l}0.915(0.052) \\
0.138(0.014) \\
0.049(0.005)\end{array}$ & $\begin{array}{l}3.907(0.224) \times 10^{13} \\
3.039(0.438) \times 10^{12} \\
1.023(0.222) \times 10^{12}\end{array}$ \\
\hline $\mathrm{HCO}^{+}$ & 89.19 & $\begin{array}{c}0.7(8.7) \\
-153.4(8.7) \\
-194.4(8.7)\end{array}$ & $\begin{array}{c}20.0^{a} \\
10.6(8.7) \\
10.0(8.7)\end{array}$ & $\begin{array}{l}-0.47096 \\
-0.18489 \\
-0.04435\end{array}$ & $\begin{array}{l}0.66217 \\
0.66217 \\
0.66217\end{array}$ & $\begin{array}{l}3.351 \\
3.351 \\
3.351\end{array}$ & $\begin{array}{l}1.242(0.058) \\
0.327(0.029) \\
0.069(0.007)\end{array}$ & $\begin{array}{l}2.936(0.747) \times 10^{13} \\
4.114(3.386) \times 10^{12} \\
8.170(0.717) \times 10^{11}\end{array}$ \\
\hline $\mathrm{HNC}$ & 90.66 & $\begin{array}{c}1.5(0.4) \\
-154.1(0.5) \\
-\end{array}$ & $\begin{array}{c}22.5(0.6) \\
8.7(1.8) \\
-\end{array}$ & $\begin{array}{c}-0.08730 \\
-0.03176 \\
-\end{array}$ & $\begin{array}{l}0.66003 \\
0.66003\end{array}$ & $\begin{array}{l}2.827 \\
2.827\end{array}$ & $\begin{array}{l}0.142(0.014) \\
0.049(0.005)\end{array}$ & $\begin{array}{l}6.061(0.641) \times 10^{12} \\
8.160(1.863) \times 10^{11}\end{array}$ \\
\hline CS & 97.98 & $\begin{array}{c}3.9(0.3) \\
-157.2(0.9)\end{array}$ & $\begin{array}{l}18.0^{a} \\
10.0^{a}\end{array}$ & $\begin{array}{l}-0.03808 \\
-0.01782\end{array}$ & $\begin{array}{l}0.64514 \\
0.64514\end{array}$ & $\begin{array}{l}1.353 \\
1.353\end{array}$ & $\begin{array}{l}0.061(0.007) \\
0.028(0.003)\end{array}$ & $\begin{array}{l}9.423(2.817) \times 10^{12} \\
2.410(1.229) \times 10^{12}\end{array}$ \\
\hline${ }^{13} \mathrm{CO}$ & 110.20 & $0.4(0.1)$ & $8.1(0.7)$ & -0.26448 & 0.54710 & 3.717 & $0.660(0.045)$ & $6.334(0.694) \times 10^{15}$ \\
\hline $\mathrm{CN}^{b}$ & 113.14 & $\begin{array}{c}0(0.1) \\
-156.0(0.1) \\
-\end{array}$ & $\begin{array}{c}14.7^{a} \\
14.7^{a} \\
-\end{array}$ & $\begin{array}{l}-0.11254^{c} \\
-0.01906^{c}\end{array}$ & $\begin{array}{l}0.62611 \\
0.62611\end{array}$ & $\begin{array}{l}3.890 \\
3.890\end{array}$ & $\begin{array}{l}0.198(0.019) \\
0.031(0.004)\end{array}$ & $\begin{array}{l}1.547(0.547) \times 10^{14} \\
2.413(0.890) \times 10^{13} \\
\quad<1.200 \times 10^{13}\end{array}$ \\
\hline
\end{tabular}

Notes. $V_{0}$ is the central velocity (local standard of rest; LSR) from a Gaussian fit, $\Delta V$ is the full width at half maximum of the Gaussian fit, $S_{1}$ corresponds to the flux density peak of the absorption line, $S_{\mathrm{c}}$ is the continuum flux density, rms is the root mean square noise level of the spectrum for a $\sim 10 \mathrm{~km} \mathrm{~s}^{-1}$ channel width, $\tau$ is the optical depth, and $N$ is the column density. ${ }^{(a)}$ Fixed parameter in the Gaussian fit. ${ }^{(b)}$ Hyperfine structure.

${ }^{(c)}$ Line intensity corresponds to the hyperfine component at $113.191 \mathrm{GHz}$.

\section{Discussion}

\subsection{Association of the absorption features with the Galactic center region}

As stated in Sect. 3.1, there are three velocity components, at $\sim 0,-153$, and $-192 \mathrm{~km} \mathrm{~s}^{-1}$ (Fig. 3). The velocity component at $0 \mathrm{~km} \mathrm{~s}^{-1}$ may be associated with gas along the line of sight across the Galactic disk as can be seen in the CO (1-0) maps from Bitran et al. (1997) and the CO (2-1) emission line maps from Sawada et al. (2001). This velocity component is the only one also detected in the ${ }^{13} \mathrm{C}$ isotopic substitutions of $\mathrm{HCO}^{+}$and $\mathrm{HCN}$, translating into a ${ }^{12} \mathrm{C} /{ }^{13} \mathrm{C}$ isotopic ratio of $65\left(\mathrm{HCO}^{+} / \mathrm{H}^{13} \mathrm{CO}^{+}\right)$and $80\left(\mathrm{HCN} / \mathrm{H}^{13} \mathrm{CN}\right)$ assuming that both transitions are optically thin. Even though Riquelme et al. (2010a) have found high ${ }^{12} \mathrm{C} /{ }^{13} \mathrm{C}$ isotopic ratios in a few specific regions in the $\mathrm{GC}$ in which gas may be infalling, the high iso- topic values found in the $0 \mathrm{~km} \mathrm{~s}^{-1}$ component are an indication that this gas represents disk gas. The isotope ratios match those found in the local ISM (70) and are much larger than the values of 20-25 usually found in the GC (Wilson 1999; Milam et al. 2005). There were no ${ }^{13} \mathrm{C}$ isotopic substitutions detected in the velocity components at -153 , and $-192 \mathrm{~km} \mathrm{~s}^{-1}$. The lower limits $(3 \sigma)$ of $\mathrm{HCO}^{+} / \mathrm{H}^{13} \mathrm{CO}^{+}$and $\mathrm{HCN} / \mathrm{H}^{13} \mathrm{CN}$ in the $-153 \mathrm{~km} \mathrm{~s}^{-1}$ component are 42 and 16, respectively. For the $-192 \mathrm{~km} \mathrm{~s}^{-1}$ component, these limits are 11 and 7. There are no foreground features expected from, for example, spiral arms at velocities of -153 and $-192 \mathrm{~km} \mathrm{~s}^{-1}$ (Bania 1977; Dame \& Thaddeus 2008). There is no $\mathrm{CO}$ emission counterpart for the velocity components at -153 and $-192 \mathrm{~km} \mathrm{~s}^{-1}$ from previous observations at the position of the quasar (Sawada et al. 2001; Bitran et al. 1997). This was also noted by Gerin \& Liszt (2017) who found HI emission around $-190 \mathrm{~km} \mathrm{~s}^{-1}$ and significantly weaker HI emis- 
Table 3. Theoretical relative intensities of the detected hyperfine transitions assuming optically thin lines and local thermodynamical equilibrium.

\begin{tabular}{cccc}
\hline \hline Molecule & $\begin{array}{c}\text { Transition } \\
\text { N'-N", J'-J", F'-F", }\end{array}$ & $\begin{array}{c}\text { Res. frequency } \\
\text { [GHz] }\end{array}$ & Relative intensity \\
\hline CN & $1-0,1 / 2-1 / 2,1 / 2-1 / 2$ & 113.12337 & 0.012 \\
& $1-0,1 / 2-1 / 2,1 / 2-3 / 2$ & 113.14415 & 0.098 \\
& $1-0,1 / 2-1 / 2,3 / 2-1 / 2$ & 113.17049 & 0.096 \\
& $1-0,1 / 2-1 / 2,3 / 2-3 / 2$ & 113.19127 & 0.125 \\
& $1-0,3 / 2-1 / 2,3 / 2-1 / 2$ & 113.48812 & 0.125 \\
& $1-0,3 / 2-1 / 2,5 / 2-3 / 2$ & 113.49097 & 0.333 \\
& $1-0,3 / 2-1 / 2,1 / 2-1 / 2$ & 113.49964 & 0.099 \\
& $1-0,3 / 2-1 / 2,3 / 2-3 / 2$ & 113.50890 & 0.096 \\
& $1-0,3 / 2-1 / 2,1 / 2-3 / 2$ & 113.52043 & 0.012 \\
\hline \multirow{H}{*}{ CO } & $1-0,3 / 2-1 / 2,2-1$ & 86.67076 & 0.420 \\
& $1-0,3 / 2-1 / 2,1-0$ & 86.70836 & 0.248 \\
& $1-0,1 / 2-1 / 2,1-1$ & 86.77746 & 0.248 \\
& $1-0,1 / 2-1 / 2,0-1$ & 86.80578 & 0.084 \\
\hline CCH & $1-0,3 / 2-1 / 2,1-1$ & 87.28410 & 0.042 \\
& $1-0,3 / 2-1 / 2,2-1$ & 87.31689 & 0.416 \\
& $1-0,3 / 2-1 / 2,1-0$ & 87.32858 & 0.208 \\
& $1-0,1 / 2-1 / 2,1-1$ & 87.40198 & 0.208 \\
& $1-0,1 / 2-1 / 2,0-1$ & 87.40716 & 0.084 \\
& $1-0,1 / 2-1 / 2,1-0$ & 87.44647 & 0.042 \\
\hline
\end{tabular}

Notes. The intensities are normalized to unity.

sion at $-150 \mathrm{~km} \mathrm{~s}^{-1}$ but $\mathrm{CO}$ emission only at $-22 \mathrm{~km} \mathrm{~s}^{-1}$ along the line of sight to the quasar. They only found $\mathrm{CO}$ emission from the bulge at $\sim-190 \mathrm{~km} \mathrm{~s}^{-1}$ in the vicinity of the quasar J1744-312 at lower Galactic latitudes $(b>-0.5)$, and the velocity component at $-150 \mathrm{~km} \mathrm{~s}^{-1}$ only in a nearby $\mathrm{CO}$ feature. This indicates that these absorption features correspond to low excitation gas that cannot be observed in emission toward the bulge of the Galaxy.

\subsection{Detected molecules}

From the 12 species observed in this study, 7 molecules were detected in the velocity component at $-153 \mathrm{~km} \mathrm{~s}^{-1}$, namely c- $\mathrm{C}_{3} \mathrm{H}_{2}, \mathrm{CCH}, \mathrm{HCN}, \mathrm{HCO}^{+}, \mathrm{HNC}, \mathrm{CS}$, and $\mathrm{CN}$. Out of those, only 3 were detected at $-192 \mathrm{~km} \mathrm{~s}^{-1}\left(\mathrm{c}-\mathrm{C}_{3} \mathrm{H}_{2}, \mathrm{HCN}\right.$ and $\left.\mathrm{HCO}^{+}\right)$.

In previous absorption studies against quasars, Lucas \& Liszt (2000a) and Liszt \& Lucas (2001) found that the molecules can be grouped into various chemical families: 1) the cyanogen- or $\mathrm{CN}$-bearing molecules, including $\mathrm{CN}, \mathrm{HCN}$, and $\mathrm{HNC}$, where the abundances of these molecules are tightly linearly correlated; 2) the $\mathrm{HCO}^{+}-\mathrm{OH}$ family, which also shows a tight linear relation (Liszt \& Lucas 1996; Lucas \& Liszt 1996); and 3) the $\mathrm{C}_{n} \mathrm{H}_{m^{-}}$ family, with a looser but still good linear correlation.

The comparison between the various families shows a high degree of correlation but a nonlinear behavior. Similar results were obtained by Godard et al. (2010) in the absorption study toward star-forming regions in a large range of Galactic longitude along the Galactic plane. Figure 4 shows the column density comparison for the family of the cyanogen molecules. We include the data from Liszt \& Lucas (2001), Godard et al. (2010), Muller et al. (2011), and the present work. We can see that our work closely follows the relationship previously established by observations of absorption against quasars at high Galactic latitude and also by observations close to the Galactic plane in star formation regions. This global trend may indicate that the chemistry that drives the cyanogen-molecule family is similar in the three different environments. The absorption lines against the quasar PKS 1830-211 from the $z=0.89$ foreground galaxy Muller et al. (2011, see the lower panel of Fig. 4) show higher column densities in all molecules by more than one order of magnitude than the other works. The green dashed line shows the fit including all data, and the continuous line shows the fit including only Galactic data (excluding the $z=0.89$ absorption against the quasar PKS 1830-211).

In Fig. 5 we plot the cyanogen-molecule family against $\mathrm{HCO}^{+}$and we find that the $\mathrm{HCO}^{+}$column density is slightly larger than in previous works when compared with $\mathrm{CN}$. We also include the recent work by Ando et al. (2016) who detected molecular absorption lines of Galactic origin toward four radio-loud quasars that were observed as bandpass and complex gain calibrators in ALMA. Figure 6 shows the column density of the $\mathrm{C}_{n} \mathrm{H}_{m}$ family against $\mathrm{HCO}^{+}$. The data set of Godard et al. (2010) was complemented by the $\mathrm{CCH}$ and $\mathrm{c}-\mathrm{C}_{3} \mathrm{H}_{2}$ data from Gerin et al. (2011). The previous works also compared the linewidth of the different molecules, but this analysis is not possible with our data because of the lower spectral resolution of our survey $\left(\sim 10 \mathrm{~km} \mathrm{~s}^{-1}\right)$. From Figs. $4-6$ we can see that all data are well correlated, which is an indication that the chemistries of the diffuse gas in the local ISM traced by the sample of Liszt \& Lucas (2001), the diffuse gas in the Galactic plane traced by Godard et al. (2010), and the diffuse gas in the Galactic bulge of this work are similar. Godard et al. (2010) showed that the column densities of the different molecules cannot be reproduced by UV-dominated chemical models (PDR models) but by dissipation of turbulent energy models. Further observations of higher rotational transitions with higher spectral resolution are needed to check our assumption of $T_{\mathrm{ex}}=T_{\mathrm{bg}}$ and to derive physical properties, such as kinetic temperature and volume density of this diffuse gas, and to compare this with chemical models.

\subsection{Mass of the low density gas and comparison with gas in emission in the $C M Z$}

Table 4 shows typical column densities along several lines of sight toward the CMZ for the detected molecules in this work. 

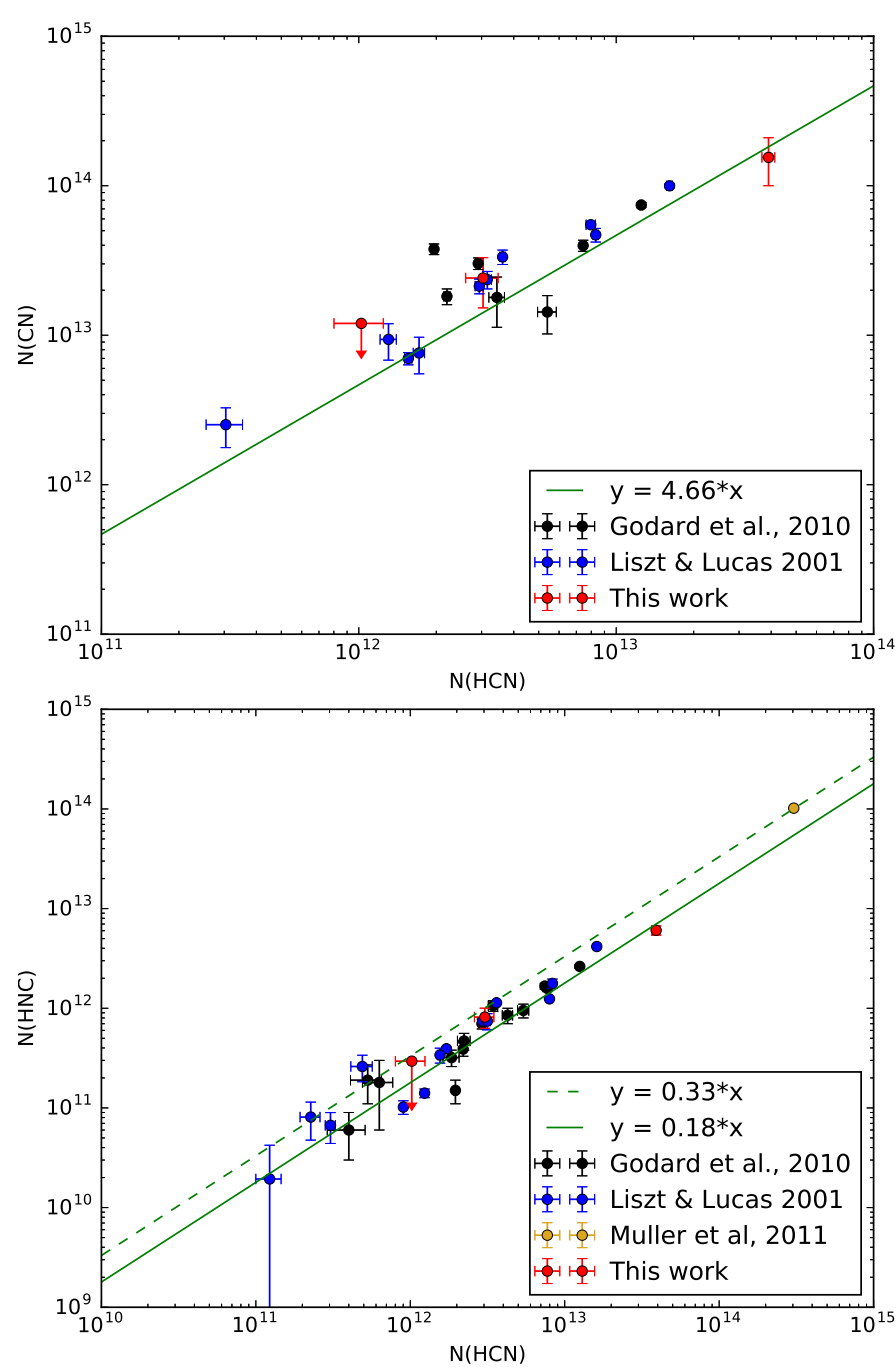

Fig. 4. Comparison of the column densities of $\mathrm{CN}, \mathrm{HCN}$, and $\mathrm{HNC}$, i.e., the cyanogen- or $\mathrm{CN}$-bearing molecular family. The black symbols correspond to the absorption study against Galactic star-forming regions by Godard et al. (2010), the blue symbols correspond to the absorption study against quasars by Lucas \& Liszt (2000a), yellow points refer to the absorption line study of a $z=0.89$ galaxy by Muller et al (2011), and the red symbols correspond to this work. The red points with highest column density correspond to the gas in the Galactic disk $\left(v \sim 0 \mathrm{~km} \mathrm{~s}^{-1}\right)$, and the red points in the middle correspond to the velocity component of $v \sim-153 \mathrm{~km} \mathrm{~s}^{-1}$. The lower red points refer to the velocity component of $v \sim-192 \mathrm{~km} \mathrm{~s}^{-1}$.

The column density of the gas detected in absorption in the bulge of the Galaxy from this work is 1-3 orders of magnitude lower than that of gas in emission in the CMZ.

To derive reliable fractional abundances for the detected species in this survey, we need to compute molecular column densities $N\left(\mathrm{H}_{2}\right)$. From the available data of this work it is not possible to derive $N\left(\mathrm{H}_{2}\right)$. However, we can estimate $N\left(\mathrm{H}_{2}\right)$ using the relation shown in Fig. 3 of Liszt \& Lucas (2001) between $\mathrm{CN}$ and $\mathrm{H}_{2}$ derived by optical observations of Federman et al. (1994). The relation between $N(\mathrm{CN})$ and $N\left(\mathrm{H}_{2}\right)$ is nonlinear with a power-law slope of 1.31. Using this relation with our data, we infer a molecular hydrogen column of $1.12 \times 10^{22} \mathrm{~cm}^{-2}$ for the potentially local gas $\left(0 \mathrm{~km} \mathrm{~s}^{-1}\right)$, $2.71 \times 10^{21} \mathrm{~cm}^{-2}$ for the velocity component at $-153 \mathrm{~km} \mathrm{~s}^{-1}$, and an upper limit $(3 \sigma)$ of $1.60 \times 10^{21} \mathrm{~cm}^{-2}$ for the velocity component at $-192 \mathrm{~km} \mathrm{~s}^{-1}$. These results are in good agreement with the recent work by Gerin \& Liszt (2017), who derived
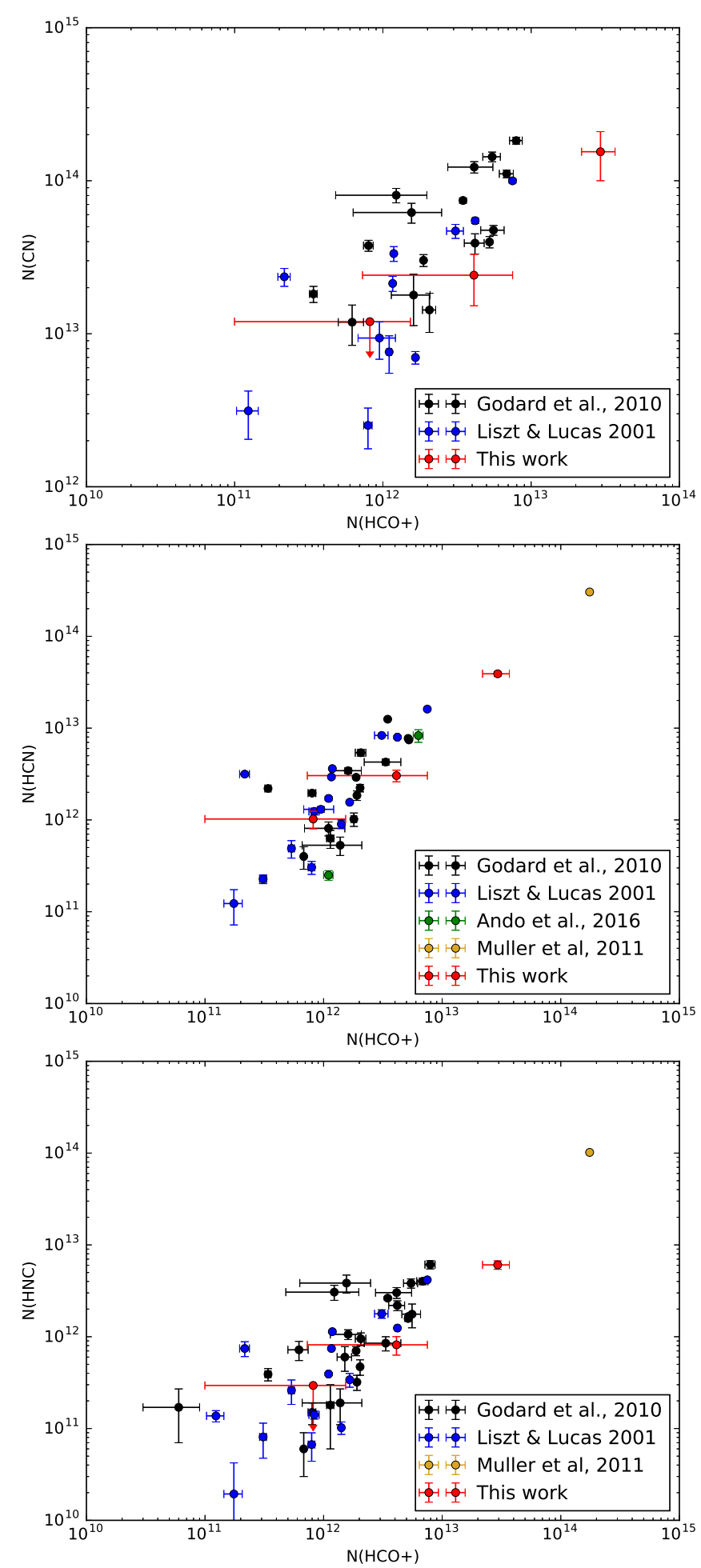

Fig. 5. Comparison of the column densities of the CN-bearing molecular family with $\mathrm{HCO}^{+}$. The black symbols correspond to the absorption study against Galactic star-forming regions by Godard et al. (2010), the blue symbols correspond to the absorption study against quasars by Lucas \& Liszt (2000a), and the red symbols refer to this work. We also include in green symbols the recent work by Ando et al. (2016) using Galactic ALMA calibrators and in yellow the absorption line study of a $z=0.89$ galaxy by Muller et al. (2011).

$N\left(\mathrm{H}_{2}\right)=1.21 \times 10^{21} \mathrm{~cm}^{-2}$ and $2.86 \times 10^{20} \mathrm{~cm}^{-2}$ for the velocity components at $\sim-150 \mathrm{~km} \mathrm{~s}^{-1}$ and $\sim-190 \mathrm{~km} \mathrm{~s}^{-1}$ respectively, using $N\left(\mathrm{HCO}^{+}\right) / N\left(\mathrm{H}_{2}\right)=3 \times 10^{-9}$ (the local value for solar 

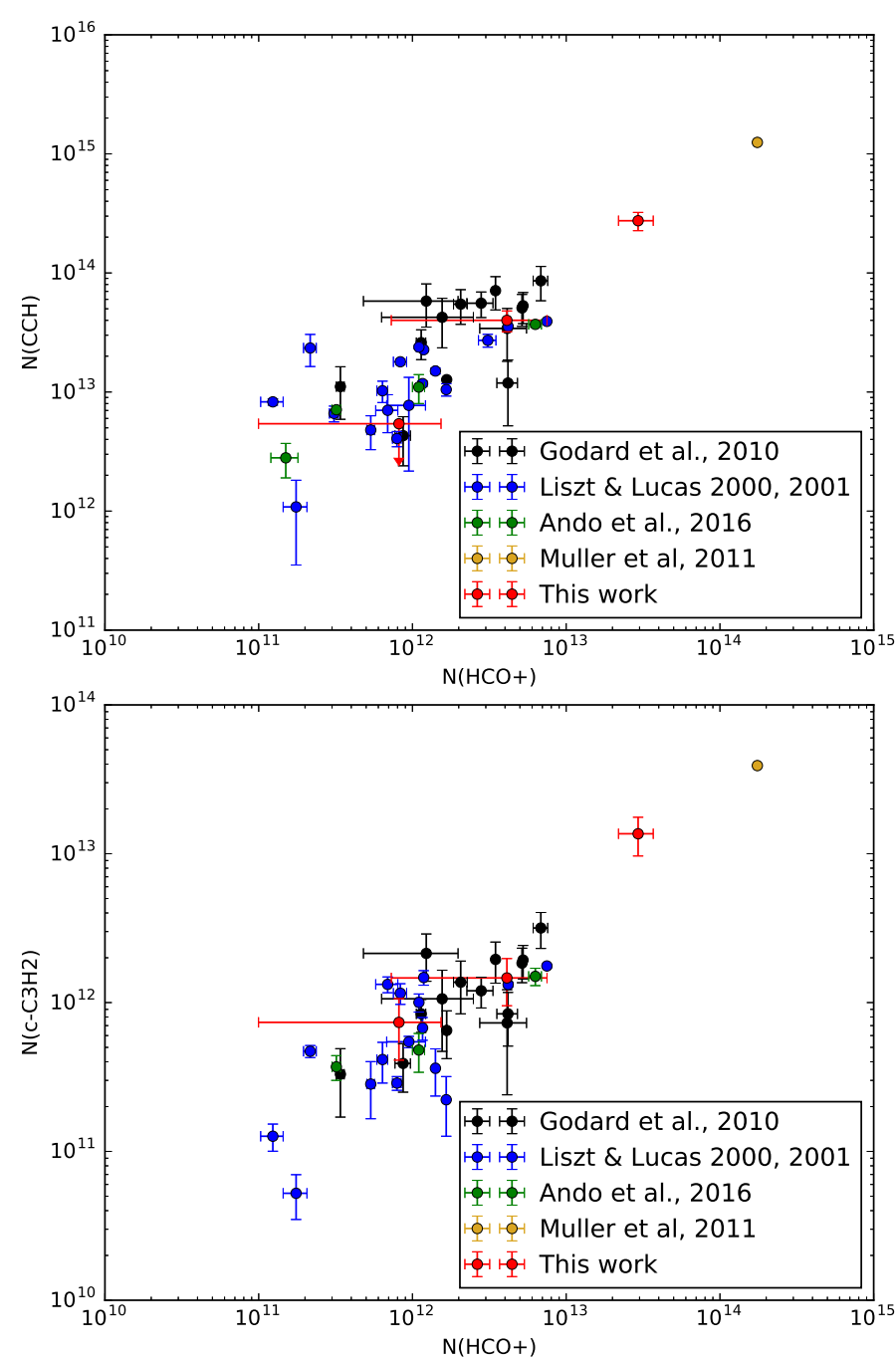

Fig. 6. Comparison of the column densities of the molecular $\mathrm{C}_{n} \mathrm{H}_{n}$ family with $\mathrm{HCO}^{+}$. The black symbols correspond to the absorption study against Galactic star-forming regions by Godard et al. (2010), the blue symbols correspond to the absorption study against quasars by Lucas \& Liszt (2000a), and the red symbols refer to this work. We also include in green symbols the work by Ando et al. (2016) and in yellow the line absorption study of the $z=0.89$ galaxy by Muller et al. (2011).

metallicity). If we use this $\mathrm{HCO}^{+}$abundance value, we derive $N\left(\mathrm{H}_{2}\right)=1.37 \times 10^{21} \mathrm{~cm}^{-2}$ and $2.72 \times 10^{20} \mathrm{~cm}^{-2}$ for the velocity components at $\sim-150 \mathrm{~km} \mathrm{~s}^{-1}$ and $\sim-190 \mathrm{~km} \mathrm{~s}^{-1}$, respectively. Riquelme et al. (2018) estimated $\mathrm{HCO}^{+}$abundances of $>3.8 \times 10^{-9}$ for several clouds in the CMZ, which yields $N\left(\mathrm{H}_{2}\right)$ similar to that derived using the nonlinear relation between $\mathrm{CN}$ and $\mathrm{H}_{2}$ and the $\mathrm{HCO}^{+}$abundance value used by Gerin \& Liszt (2017).

We can obtain a very rough estimate of the total mass of the low density gas using the column density of the velocity component at $-153 \mathrm{~km} \mathrm{~s}^{-1}$. Since the quasar is located at $(l, b)=\left(-2.13,-1^{\circ}\right)$, we assume that the low density gas has, at least, an extension of $4^{\circ} .3$ in Galactic longitude if this component were symmetrically distributed ${ }^{5}$ and showed a spherical

\footnotetext{
5 This is not actually true for the molecular gas seen in emission in the CMZ. The CMZ shows an asymmetry in the distribution of the molecular gas with two-thirds of the dense gas being placed at positive longitudes (Morris \& Serabyn 1996), while our source is located at negative longitude.
}

geometry with a radius equal to the projected distance between our source and the GC. Considering a distance of $8.5 \mathrm{kpc}$ for the $\mathrm{GC}$, the total mass of the diffuse component could be as high as $1.9 \times 10^{7} M_{\odot}$. In the literature there are many independent estimations of the total gas mass for the GC (see, Ferrière et al. 2007, for a review). For example, Launhardt et al. (2002) derived a total mass of the interstellar hydrogen for the entire CMZ of $\sim 6 \times 10^{7} M_{\odot}$. The molecular gas observed in emission in the CMZ shows different components, one dense and cool $\left(T_{\text {kin }}<50 \mathrm{~K}, n \sim 10^{3.5} \mathrm{~cm}^{-3}\right)$, and the $n \sim 10^{4} \mathrm{~cm}^{-3}, T_{\text {kin }}>$ $50 \mathrm{~K}$ component of Ao et al. (2013); Ginsburg et al. (2016) and also a warmer and thinner component with kinetic temperature $\sim 150 \mathrm{~K}$ and $n \sim 10^{2.5} \mathrm{~cm}^{-3}$. Dahmen et al. (1998) estimated $1.2-6.4 \times 10^{7} M_{\odot}$ for the dense gas and $0.7-1.4 \times 10^{7} M_{\odot}$ for the thin gas for the central $\sim 600 \mathrm{pc}$ of the GC. Although we only have one line of sight, this result may indicate that the low density gas found in this work represents a considerable fraction of about one-third of the total gas mass in the GC.

\subsection{Origin of the excitation of the diffuse gas in the bulge and its relationship with the $\mathrm{CMZ}$}

There are still many unknown parameters in the low excitation absorption gas found in this work, referring to its relationship with the CMZ and the association with the warm low density component found by infrared absorption spectroscopy of $\mathrm{H}_{3}^{+}$and CO by Oka et al. (2005), Goto et al. (2008), and Geballe \& Oka (2010). The increased amount of sightlines available for absorption studies in $\mathrm{H}_{3}^{+}$and $\mathrm{CO}$ (Geballe et al. 2015) show that the warm, low density gas fills a large percentage of the volume of the CMZ, but the sightlines do not cover regions outside the $\mathrm{CMZ}$ associated with the bulge of the Galaxy.

Goto et al. (2014) observed the $\mathrm{H}_{3}^{+}$and ro-vibrational transitions of $\mathrm{CO}$ on sightlines toward two luminous infrared sources located in the central stellar cluster associated with the GC and found that the absorption occurs in three kinds of gaseous environments: 1) cold dense and diffuse gas associated with foreground spiral arms containing both species $\left(\mathrm{H}_{3}^{+}\right.$and $\left.\mathrm{CO}\right)$; 2) warm and diffuse gas absorbing over a wide and mostly negative velocity range $\left(-180\right.$ to $\left.+20 \mathrm{~km} \mathrm{~s}^{-1}\right)$, which may be filling a large portion of the $\mathrm{CMZ}$, containing $\mathrm{H}_{3}^{+}$and little $\mathrm{CO}$; and 3 ) warm, dense, and compact clouds that contain both species. With respect to the second kind of gas, Goto et al. (2014) and Goto et al. (2015) relate the large negative velocities ( -160 to $-110 \mathrm{~km} \mathrm{~s}^{-1}$ ) to the expanding molecular ring (EMR; Scoville 1972), which may be expanding from the GC with a radial velocity of $\sim 200 \mathrm{~km} \mathrm{~s}^{-1}$. As examples of the third kind of gas, Goto et al. (2014) find absorption along the lines of sight toward the central cluster at velocities $\sim 50 \mathrm{~km} \mathrm{~s}^{-1}$, which may be associated with an inward extension of the circunnuclear disk. Referring to the clouds detected in this work, their relationship with the diffuse and warm gas in the CMZ is not clear. Also, not settled are the questions of whether these clouds are associated with features seen in emission in the GC region, such as EMR, or if they are warm, dense and compact clouds, similar to the third kind of environment discussed above, which is unlikely since they could also be detected in emission. Higher rotational transitions of the detected molecules are needed to derive the physical properties of the detected clouds and infrared absorption studies toward larger regions of the GC to unveil the extension of the warm and diffuse gas found by Oka et al. (2005). 
D. Riquelme et al.: The diffuse molecular component in the nuclear bulge of the Milky Way

Table 4. Column densities for the detected species (in emission) in the GC along various lines of sight toward the CMZ from recent previous works.

\begin{tabular}{ccccccc}
\hline \hline Molecule & $\begin{array}{c}\mathrm{SgrB2N}^{a} \\
{\left[\mathrm{~cm}^{-2}\right]}\end{array}$ & $\begin{array}{c}\mathrm{SgrB}^{2} \mathrm{M}^{a} \\
{\left[\mathrm{~cm}^{-2}\right]}\end{array}$ & $\begin{array}{c}\mathrm{CND}^{b} \\
{\left[\mathrm{~cm}^{-2}\right]}\end{array}$ & $\begin{array}{c}\text { LOS+0.693 } \\
{\left[\mathrm{cm}^{-2}\right]}\end{array}$ & $\begin{array}{c}\mathrm{LOS}^{c}-0.11^{c} \\
{\left[\mathrm{~cm}^{-2}\right]}\end{array}$ & $\begin{array}{c}\mathrm{CMZ}^{d, e} \\
{\left[\mathrm{~cm}^{-2}\right]}\end{array}$ \\
\hline $\mathrm{c}^{-} \mathrm{C}_{3} \mathrm{H}_{2}$ & $2.50 \times 10^{14}$ & $2.60 \times 10^{14}$ & & $9.4 \pm 2.5 \times 10^{13}$ & $6.0 \pm 0.7 \times 10^{13}$ \\
$\mathrm{CCH}$ & $2.00 \times 10^{15}$ & $5.00 \times 10^{15}$ & & $3.162 \pm 0.186 \times 10^{15}$ & $2.512 \pm 0.24 \times 10^{15}$ & $>4.80 \times 10^{15}$ \\
$\mathrm{HCN}$ & $1.07 \times 10^{17}$ & $1.79 \times 10^{16}$ & $3.38 \pm 0.40 \times 10^{15}$ & $>2.40 \times 10^{15}$ & $1.7 \times 10^{15}$ \\
$\mathrm{HCO}^{+}$ & $5.07 \times 10^{15}$ & $7.45 \times 10^{15}$ & $5.26 \pm 1.28 \times 10^{14}$ & $5.70 \pm 0.13 \times 10^{14}$ & $3.6 \pm 0.08 \times 10^{14}$ & $5.0 \times 10^{14}$ \\
$\mathrm{HNC}$ & $4.79 \times 10^{15}$ & $5.98 \times 10^{15}$ & $1.90 \pm 0.29 \times 10^{14}$ & $>2.4 \times 10^{15}$ & $>1.8 \times 10^{15}$ & $7.0 \times 10^{14}$ \\
$\mathrm{CS}$ & $2.59 \times 10^{17}$ & $1.49 \times 10^{16}$ & $1.07 \pm 0.13 \times 10^{15}$ & & & \\
${ }^{13} \mathrm{CO}$ & $3.08 \times 10^{18}$ & $3.45 \times 10^{18}$ & & $2.81 \pm 0.0644 \times 10^{17}$ & $1.15 \pm 0.095 \times 10^{17}$ \\
$\mathrm{CN}$ & $6.93 \times 10^{14}$ & $9.90 \times 10^{15}$ & $5.48 \pm 3.75 \times 10^{15}$ & $1.698 \pm 0.174 \times 10^{15}$ & $1.738 \pm 0.195 \times 10^{15}$ \\
\hline
\end{tabular}

Notes. ${ }^{(a)}$ Belloche et al. (2013) of the velocity component closest to the systemic velocity of Sgr B2N (64 km s$\left.{ }^{-1}\right)$ and Sgr B2M (62 km s$\left.{ }^{-1}\right)$; (b) Harada et al. (2015); ${ }^{(c)}$ Armijos-Abendaño et al. (2015); ${ }^{(d)}$ Jones et al. (2012); ${ }^{(e)}$ average value over the CMZ corrected for optical depth effects.

\subsection{A new line of sight for Galactic diffuse ISM studies}

The velocity component at $v \sim 0 \mathrm{~km} \mathrm{~s}^{-1}$ adds a new line of sight for Galactic diffuse ISM studies. At the moment, there is only a limited number of Galactic absorption systems known in the literature $(\sim 30)$ and only few of these systems have been extensively studied (Lucas \& Liszt 1996, 2000a; Liszt \& Lucas 2001; Liszt et al. 2014). Recently, Ando et al. (2016) using calibrators in the ALMA Archive found four absorption systems of Galactic origin (three of these are new detections). In this work, the detected absorption against the quasar J1744-312 at the velocity of $v \sim 0 \mathrm{~km} \mathrm{~s}^{-1}$ is of Galactic origin, likely coming from local gas or gas belonging to the spiral arms. Radial components of rotation velocities collapse to zero in the direction of the Galactic center. This velocity component shows a rich chemistry with the detection of the 12 species listed in Table 2, and in general, its column density is about one order of magnitude higher than the velocity components corresponding to the bulge components with highly negative velocities. From these species, the detection of $\mathrm{HCO}$ at the velocity component $\sim 0 \mathrm{~km} \mathrm{~s}^{-1}$ is particularly remarkable, which is the sixth detection in absorption found up to date in the diffuse medium (Liszt et al. 2014; Ando et al. 2016). HCO is a PDR tracer even in the diffuse medium $\left(n_{\mathrm{H}_{2}} \sim 10^{1}-10^{2} \mathrm{~cm}^{-3}\right)$, and its detection is the result of the ionization of carbon $\left(\mathrm{C}^{+}\right)$by distant OB stars, which together with $\mathrm{H}_{2}$ lead to the formation of $\mathrm{CH}_{2}$, and then $\mathrm{HCO}$, probably in the gas phase through the reaction $\mathrm{O}+\mathrm{CH}_{2} \rightarrow \mathrm{HCO}+\mathrm{H}$ (Gerin et al. 2009). In this work, the column density ratio $N(\mathrm{HCO}) / N\left(\mathrm{H}^{13} \mathrm{CO}^{+}\right)$commonly used as diagnostic of the presence of far-UV radiation field has a value of 9, which is higher than 1 (the threshold for a PDR-like environment; Gerin et al. 2009), but lower than the values derived from previous absorption studies (19.3) and toward well-known PDRs.

We also detected $\mathrm{SiO}$ at the velocity component $\sim 0 \mathrm{~km} \mathrm{~s}^{-1}$ with a low fractional abundance of $N(\mathrm{SiO}) / N\left(\mathrm{H}_{2}\right)$ of $1 \times 10^{-10}$ consistent with the diffuse and translucent clouds studied using extragalactic continuum sources by Lucas \& Liszt (2000b). $\mathrm{SiO}$ has been extensively studied in the molecular clouds of the GC region finding high abundances associated with large scale shocks (see, e.g., Martín-Pintado et al. 1997; Hüttemeister et al. 1998; Riquelme et al. 2010b; Minh et al. 2015; Tsuboi et al. 2015, among others), and its enhancement in the disk has been associated with outflows. $\mathrm{SiO}$ can also be formed without a shock at a low abundance level as shown by previous detections in photon dominated regions (Schilke et al. 2001) and in diffuse gas (Lucas \& Liszt 2000b), and its underabundance in our case could be due to a high degree of depletion of Si from the gas phase.

\section{Conclusions}

Using ALMA in the $3 \mathrm{~mm}$ wavelength range, we detected a low excitation gas component related to the bulge of the Galaxy, seen in absorption against the background quasar J1744-312. In addition, we also found near zero velocity absorption lines that may originate from the Galactic disk.

If we assume that the derived column density from the bulge is uniform over the volume subtended by the source, we tentatively estimate that the amount of this low excitation gas in the bulge may be as high as $1.9 \times 10^{7} M_{\odot}$, which in such a scenario would correspond to a fraction of one-third of the total gas of the CMZ.

The comparison of the column densities derived in this work with previous studies indicate that the chemistry of the diffuse gas in the local ISM, Galactic plane, and bulge of the Galaxy may be similar. This result needs to be confirmed by detailed observations of higher rotational transitions with higher spectral resolution to derive reliable column densities and physical properties of this gas.

Acknowledgements. This work was carried out within the Collaborative Research Council 956, subproject A5, funded by the Deutsche Forschungsgemeinschaft (DFG). L.B. and R.F. acknowledge support by CONICYT CATA-Basal project PFB-06. We thank Arnaud Belloche for useful discussions. We thank Holger S. P. Müller for providing the $\mathrm{HCO}$ partition function value for $2.725 \mathrm{~K}$. We thank the anonymous referee for critical reading and constructive comments that helped to improve this manuscript.

\section{References}

Ando, R., Kohno, K., Tamura, Y., et al. 2016, PASJ, 68, 6

Ao, Y., Henkel, C., Menten, K. M., et al. 2013, A\&A, 550, A135

Armijos-Abendaño, J., Martín-Pintado, J., Requena-Torres, M. A., Martín, S., \& Rodríguez-Franco, A. 2015, MNRAS, 446, 3842

Bania, T. M. 1977, ApJ, 216, 381

Belloche, A., Müller, H. S. P., Menten, K. M., Schilke, P., \& Comito, C. 2013, A\&A, 559, A47

Bitran, M., Alvarez, H., Bronfman, L., May, J., \& Thaddeus, P. 1997, A\&AS, 125,99

Caton, W. M., Mannella, G. G., Kalaghan, P. M., Barrington, A. E., \& Ewen, H. I. 1968, ApJ, 151, L153

Charlot, P., Boboltz, D. A., Fey, A. L., et al. 2010, AJ, 139, 1713 
Dahmen, G., Huttemeister, S., Wilson, T. L., \& Mauersberger, R. 1998, A\&A, 331,959

Dame, T. M., \& Thaddeus, P. 2008, ApJ, 683, L143

Federman, S. R., Strom, C. J., Lambert, D. L., et al. 1994, ApJ, 424, 772

Ferrière, K., Gillard, W., \& Jean, P. 2007, A\&A, 467, 611

Geballe, T. R., \& Oka, T. 2010, ApJ, 709, L70

Geballe, T. R., Oka, T., Goto, M., Indriolo, N., \& McCall, B. J. 2011, The Molecular Universe, eds. J. Cernicharo \& R. Bachiller, in IAU Symp., 280

Geballe, T. R., Oka, T., Lambrides, E., Yeh, S., \& Goto, M. 2015, in Amer. Astron. Soc. Meet. Abstr., 225, 142.06

Gerin, M., \& Liszt, H. 2017, A\&A, 600, A48

Gerin, M., Goicoechea, J. R., Pety, J., \& Hily-Blant, P. 2009, A\&A, 494, 977

Gerin, M., Kaźmierczak, M., Jastrzebska, M., et al. 2011, A\&A, 525, A116

Ginsburg, A., Henkel, C., Ao, Y., et al. 2016, A\&A, 586, A50

Godard, B., Falgarone, E., Gerin, M., Hily-Blant, P., \& de Luca, M. 2010, A\&A, 520, A20

Goto, M., McCall, B. J., Geballe, T. R., et al. 2002, PASJ, 54, 951

Goto, M., Usuda, T., Nagata, T., et al. 2008, ApJ, 688, 306

Goto, M., Geballe, T. R., Indriolo, N., et al. 2014, ApJ, 786, 96

Goto, M., Geballe, T. R., Usuda, T., et al. 2015, in AIP Conf. Ser., 1642, 377

Harada, N., Riquelme, D., Viti, S., et al. 2015, A\&A, 584, A102

Henkel, C., Braatz, J. A., Menten, K. M., \& Ott, J. 2008, A\&A, 485, 451

Hüttemeister, S., Wilson, T. L., Bania, T. M., \& Martin-Pintado, J. 1993, A\&A, 280,255

Hüttemeister, S., Dahmen, G., Mauersberger, R., et al. 1998, A\&A, 334, 646 Jones, P. A., Burton, M. G., Cunningham, M. R., et al. 2012, MNRAS, 419, 296

Launhardt, R., Zylka, R., \& Mezger, P. G. 2002, A\&A, 384, 112

Lazio, T. J. W., \& Cordes, J. M. 1998, ApJ, 505, 715

Le Petit, F., Ruaud, M., Bron, E., et al. 2016, A\&A, 585, A105

Linke, R. A., Stark, A. A., \& Frerking, M. A. 1981, ApJ, 243, 147

Liszt, H., \& Lucas, R. 1996, A\&A, 314, 917

Liszt, H., \& Lucas, R. 2001, A\&A, 370, 576

Liszt, H. S., Pety, J., \& Lucas, R. 2008, A\&A, 486, 493

Liszt, H. S., Pety, J., Gerin, M., \& Lucas, R. 2014, A\&A, 564, A64

Lucas, R., \& Liszt, H. 1996, A\&A, 307, 237

Lucas, R., \& Liszt, H. S. 2000a, A\&A, 358, 1069

Lucas, R., \& Liszt, H. S. 2000b, A\&A, 355, 327

Martín-Pintado, J., de Vicente, P., Fuente, A., \& Planesas, P. 1997, ApJ, 482, L45
Menten, K. M., Carilli, C. L., \& Reid, M. J. 1999, in Highly Redshifted Radio Lines, eds. C. L. Carilli, S. J. E. Radford, K. M. Menten, \& G. I. Langston, ASP Conf. Ser., 156, 218

Menten, K. M., Güsten, R., Leurini, S., et al. 2008, A\&A, 492, 725

Milam, S. N., Savage, C., Brewster, M. A., Ziurys, L. M., \& Wyckoff, S. 2005, ApJ, 634, 1126

Minh, Y. C., Liu, H. B., Su, Y.-N., et al. 2015, ApJ, 808, 86

Morris, M., \& Serabyn, E. 1996, ARA\&A, 34, 645

Müller, H. S. P., Thorwirth, S., Roth, D. A., \& Winnewisser, G. 2001, A\&A, 370, L49

Müller, H. S. P., Schlöder, F., Stutzki, J., \& Winnewisser, G. 2005, J. Mol. Struct., 742,215

Muller, S., Beelen, A., Guélin, M., et al. 2011, A\&A, 535, A103

Muller, S., Combes, F., Guélin, M., et al. 2014, A\&A, 566, A112

Muller, S., Müller, H. S. P., Black, J. H., et al. 2016, A\&A, 595, A128

Nikolic, B., Bolton, R. C., Graves, S. F., Hills, R. E., \& Richer, J. S. 2013, A\&A, 552, A104

Nyman, L.-A. 1984, A\&A, 141, 323

Oka, T., Geballe, T. R., Goto, M., Usuda, T., \& McCall, B. J. 2005, ApJ, 632, 882

Pickett, H. M., Poynter, R. L., Cohen, E. A., et al. 1998, J. Quant. Spectr. Rad. Transf., 60, 883

Riquelme, D., Amo-Baladrón, M. A., Martín-Pintado, J., et al. 2010a, A\&A, 523, A51

Riquelme, D., Bronfman, L., Mauersberger, R., May, J., \& Wilson, T. L. 2010b, A\&A, 523, A45

Riquelme, D., Amo-Baladron, A., Martin-Pintado, J., et al. 2018, A\&A, in press, DOI: $10.1051 / 0004-6361 / 201629065$

Rupen, M. P. 1999, in Synthesis Imaging in Radio Astronomy II, eds. G. B. Taylor, C. L. Carilli, \& R. A. Perley, ASP Conf. Ser., 180, 229

Sawada, T., Hasegawa, T., Handa, T., et al. 2001, ApJS, 136, 189

Schilke, P., Pineau des Forêts, G., Walmsley, C. M., \& Martín-Pintado, J. 2001, A\&A, 372, 291

Scoville, N. Z. 1972, ApJ, 175, L127

Shimabukuro, F. I., \& Wilson, W. J. 1973, J. Geophys. Res., 78, 6136

Tsuboi, M., Miyazaki, A., \& Uehara, K. 2015, PASJ, 67, 90

Wilson, T. L. 1999, Rep. Progr. Phys., 62, 143

Wilson, T. L., Henkel, C., \& Hüttemeister, S. 2006, A\&A, 460, 533 
D. Riquelme et al.: The diffuse molecular component in the nuclear bulge of the Milky Way

\section{Appendix A: Complementary tables and figures}

Table A.1. Molecular data for detected transitions.

\begin{tabular}{|c|c|c|c|c|c|c|c|}
\hline Molecule & Transitions & $\begin{array}{c}\text { Frequency } \\
{[\mathrm{GHz}]}\end{array}$ & $\begin{array}{c}\mu \\
\text { [Debye] }\end{array}$ & $Q_{2.73}$ & $\begin{array}{c}A_{u l} \\
{\left[\mathrm{~s}^{-1}\right]}\end{array}$ & $\begin{array}{c}E_{l} \\
{\left[\mathrm{~cm}^{-1}\right]}\end{array}$ & Reference \\
\hline $\mathrm{c}-\mathrm{C}_{3} \mathrm{H}_{2}$ & $22_{1,2}-1_{0,1}$ & 85.338894 & 3.27 & $8.8732^{b}$ & $2.3221 \times 10^{-5}$ & 1.6332 & CDMS \\
\hline $\mathrm{H}^{13} \mathrm{CN}$ & $1-0, F=1-1,2-1,0-1$ & 86.339921 & 2.9852 & 5.1261 & $2.2255 \times 10^{-5}$ & 0.0 & CDMS \\
\hline $\mathrm{HCO}$ & $1_{0,1}-0_{0,0}, J=3 / 2-1 / 2, F=2-1$ & 86.67076 & $\mu_{a}=1.3626, \mu_{b}=0.700$ & $6.7785^{c}$ & $4.6889 \times 10^{-6}$ & 0.0129 & JPL \\
\hline $\mathrm{HCO}$ & $1_{0,1}-0_{0,0}, J=3 / 2-1 / 2, F=1-0$ & 86.70836 & $\mu_{a}=1.3626, \mu_{b}=0.700$ & $6.7785^{c}$ & $4.5951 \times 10^{-6}$ & 0.0000 & JPL \\
\hline $\mathrm{H}^{13} \mathrm{CO}^{+}$ & $1-0$ & 86.7542884 & 3.90 & 1.7028 & $3.8535 \times 10^{-5}$ & 0.0 & CDMS \\
\hline $\mathrm{HCO}$ & $1_{0,1}-0_{0,0}, J=1 / 2-1 / 2, F=1-1$ & 86.77746 & $\mu_{a}=1.3626, \mu_{b}=0.700$ & $6.7785^{c}$ & $4.6065 \times 10^{-6}$ & 0.0129 & JPL \\
\hline $\mathrm{HCO}$ & $1_{0,1}-0_{0,0}, J=1 / 2-1 / 2, F=0-1$ & 86.80578 & $\mu_{a}=1.3626, \mu_{b}=0.700$ & $6.7785^{c}$ & $4.7124 \times 10^{-6}$ & 0.0129 & JPL \\
\hline $\mathrm{SiO}$ & $v=0,2-1$ & 86.846985 & 3.0882 & 2.9760 & $2.9274 \times 10^{-5}$ & 1.4485 & CDMS \\
\hline $\mathrm{CCH}$ & $N=1-0, J=3 / 2-1 / 2, F=1-1$ & 87.284105 & 0.770 & 6.7735 & $2.5989 \times 10^{-7}$ & 0.0015 & CDMS \\
\hline $\mathrm{CCH}$ & $N=1-0, J=3 / 2-1 / 2, F=2-1$ & 87.316898 & 0.770 & 6.7735 & $1.5314 \times 10^{-6}$ & 0.0015 & CDMS \\
\hline $\mathrm{CCH}$ & $N=1-0, J=3 / 2-1 / 2, F=1-0$ & 87.328585 & 0.770 & 6.7735 & $1.2718 \times 10^{-6}$ & 0.0 & CDMS \\
\hline $\mathrm{CCH}$ & $N=1-0, J=1 / 2-1 / 2, F=1-1$ & 87.401989 & 0.770 & 6.7735 & $1.2749 \times 10^{-6}$ & 0.0015 & CDMS \\
\hline $\mathrm{CCH}$ & $N=1-0, J=1 / 2-1 / 2, F=0-1$ & 87.407165 & 0.770 & 6.7735 & $1.5361 \times 10^{-6}$ & 0.0015 & CDMS \\
\hline $\mathrm{CCH}$ & $N=1-0, J=1 / 2-1 / 2, F=1-0$ & 87.446470 & 0.770 & 6.7735 & $2.6133 \times 10^{-7}$ & 0.0 & CDMS \\
\hline $\mathrm{HCN}$ & $1-0$ & 88.6316022 & 2.9852 & 5.03 & $2.4074 \times 10^{-5}$ & 0.0 & CDMS \\
\hline $\mathrm{HCO}^{+}$ & $1-0$ & 89.1885247 & 3.90 & 1.6691 & $4.1869 \times 10^{-5}$ & 0.0 & CDMS \\
\hline $\mathrm{HNC}$ & $1-0$ & 90.6635680 & 3.05 & 1.6497 & $2.6896 \times 10^{-5}$ & 0.0 & CDMS \\
\hline CS & $2-1$ & 97.9809533 & 1.958 & 2.6827 & $1.6792 \times 10^{-5}$ & 1.6342 & CDMS \\
\hline${ }^{13} \mathrm{CO}$ & $1-0$ & 110.2013543 & 0.11046 & 2.8912 & $6.3331 \times 10^{-8}$ & 0.0 & CDMS \\
\hline \multirow[t]{7}{*}{$\mathrm{CN}$} & $1-01 / 2-1 / 2 F=1 / 2-3 / 2$ & 113.1441573 & 1.45 & 8.5178 & $1.053 \times 10^{-5}$ & 0.0 & CDMS \\
\hline & $1-01 / 2-1 / 2 F=3 / 2-1 / 2$ & 113.1704915 & & 8.5178 & $5.1451 \times 10^{-6}$ & 0.0007 & CDMS \\
\hline & $1-01 / 2-1 / 2 F=3 / 2-3 / 2$ & $113.1912787^{a}$ & & 8.5178 & $6.683 \times 10^{-6}$ & 0.0 & CDMS \\
\hline & $1-03 / 2-1 / 2 F=3 / 2-1 / 2$ & 113.4881202 & & 8.5178 & $6.7363 \times 10^{-6}$ & 0.0007 & CDMS \\
\hline & $1-03 / 2-1 / 2 F=5 / 2-3 / 2$ & 113.4909702 & & 8.5178 & $1.1924 \times 10^{-5}$ & 0.0 & CDMS \\
\hline & $1-03 / 2-1 / 2 F=1 / 2-1 / 2$ & 113.4996443 & & 8.5178 & $1.0629 \times 10^{-5}$ & 0.0007 & CDMS \\
\hline & $1-03 / 2-1 / 2 F=3 / 2-3 / 2$ & 113.5089074 & & 8.5178 & $5.1904 \times 10^{-6}$ & 0.0 & CDMS \\
\hline
\end{tabular}

Notes. $\mu$ corresponds to the dipole moment along the molecular axis. $Q_{2.73}$ is the partition function at $T_{\mathrm{ex}}=2.73 \mathrm{~K}$. $A_{u l}$ is the Einstein coefficient. $\mathrm{E}_{l}$ is the energy of the lower level. ${ }^{(a)}$ Line used to estimate the optical depth (see Sect. 3.1). ${ }^{(b)}$ Partition function for ortho c-C3H2. ${ }^{(c)}$ Holger S. P. Muller (priv. comm.). 
A\&A 610, A43 (2018)
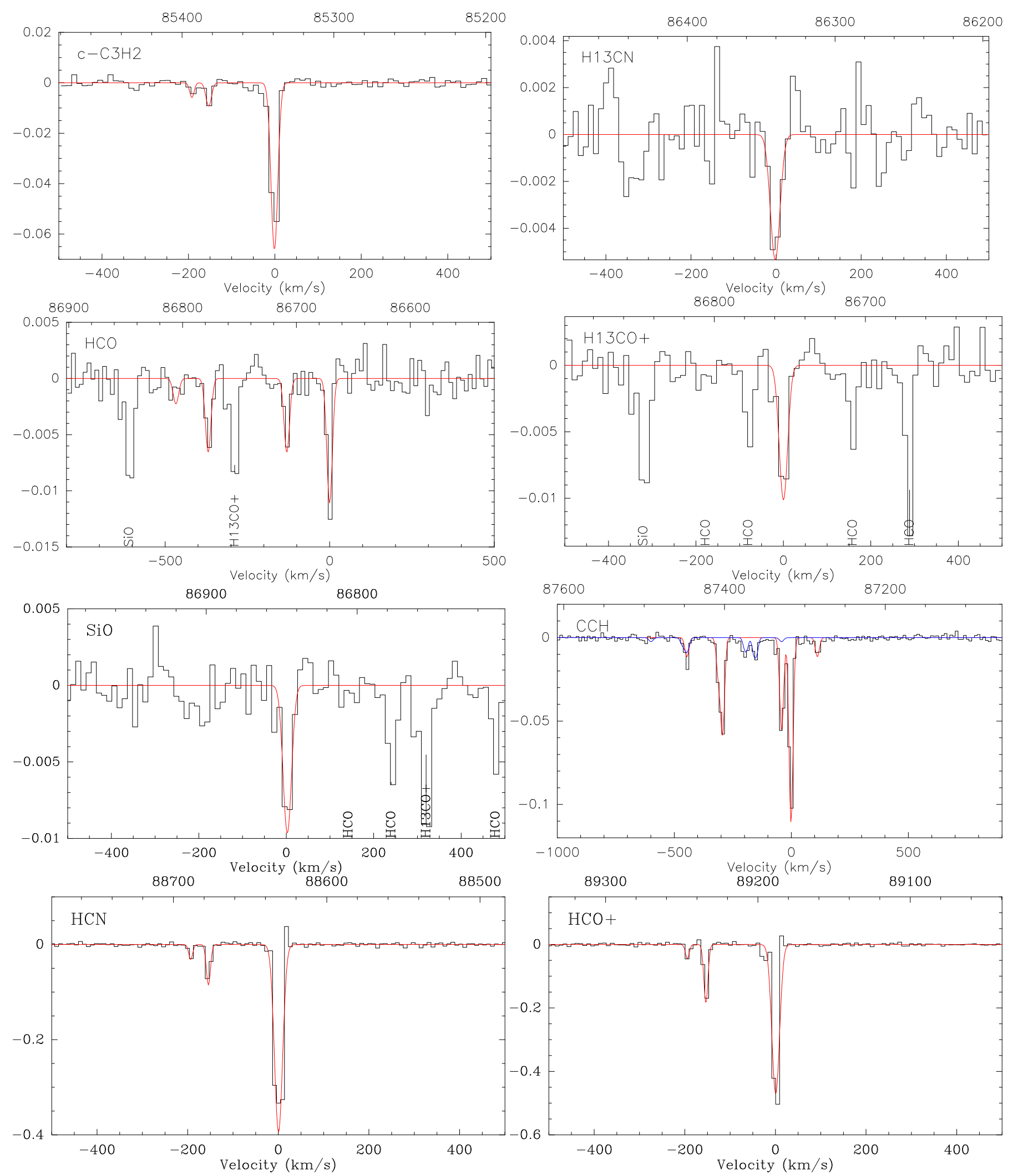

Fig. A.1. Survey. In red: Gaussian fit for each detected species. For the species with HFS resolved (CCH, HCO, CN), the red line shows the velocity component at $\sim 0 \mathrm{~km} \mathrm{~s}^{-1}$ and the blue line shows the velocity component at $\sim-153 \mathrm{~km} \mathrm{~s}^{-1}$. 
D. Riquelme et al.: The diffuse molecular component in the nuclear bulge of the Milky Way
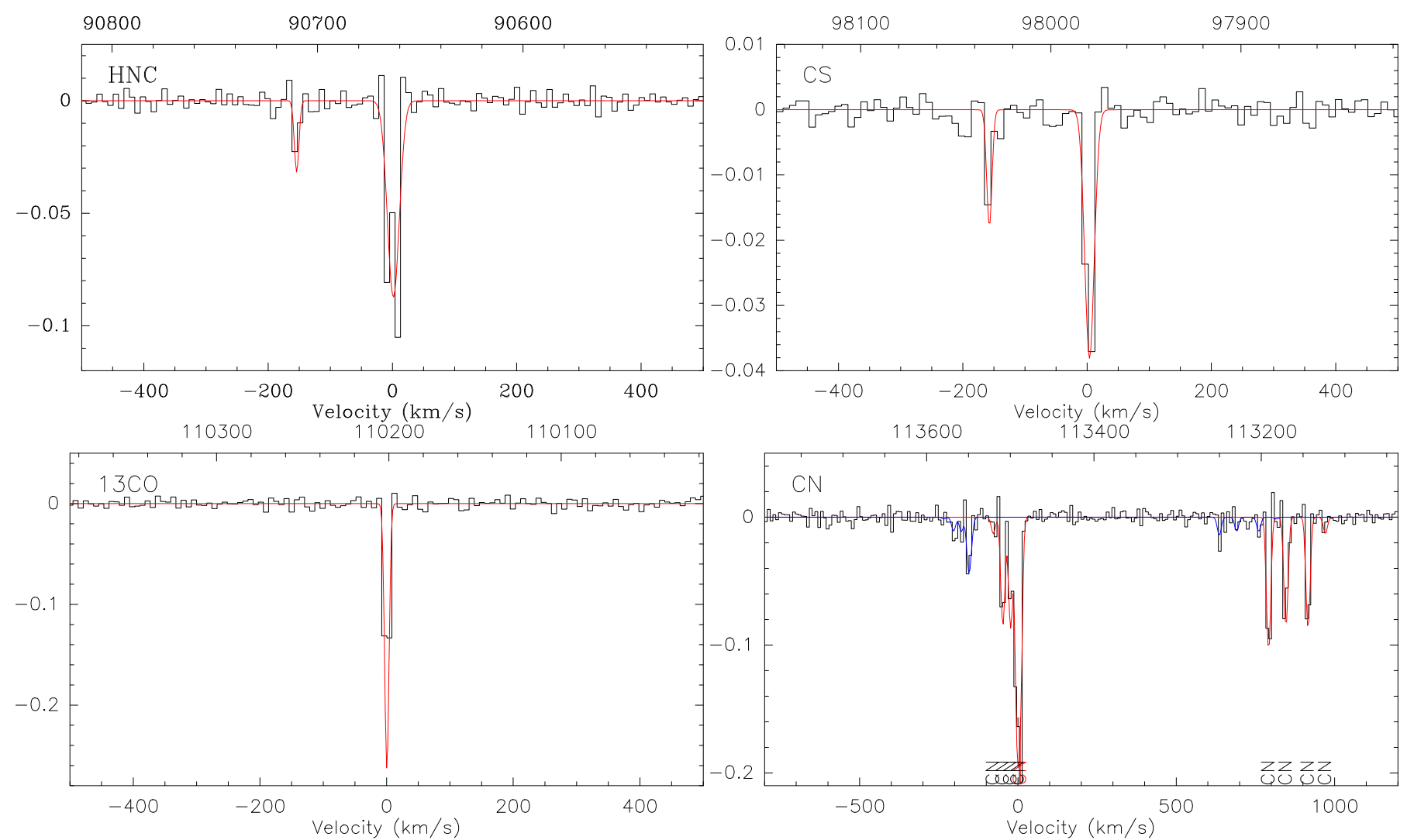

Fig. A.2. Survey. In red: Gaussian fit for each detected species. For the species with HFS resolved (CCH, HCO, CN), the red line shows the velocity component at $\sim 0 \mathrm{~km} \mathrm{~s}^{-1}$ and the blue line shows the velocity component at $\sim-153 \mathrm{~km} \mathrm{~s}^{-1}$. 